\title{
Improving the accuracy of cardiac DTI by averaging the complex data
}

\author{
Andrew D Scott ${ }^{1,2^{*}}$, Sonia Nielles-Vallespin ${ }^{3}$, Pedro Ferreira ${ }^{1,2}$, Laura-Ann McGill ${ }^{2,1}$, Dudley J Pennell ${ }^{1,2}$, \\ David Firmin ${ }^{1,2}$ \\ From 18th Annual SCMR Scientific Sessions \\ Nice, France. 4-7 February 2015
}

\section{Background}

When performing cardiac diffusion tensor imaging (cDTI) multiple averages are typically acquired to compensate for the low signal to noise ratio of the individual images. However, the potential for reducing noise in low signal areas is not fully realized when the averaging is performed on the magnitude data[1]. Averaging the complex cDTI data is not straightforward as the diffusion weighting introduces a different, spatially varying phase across each image. In this work we use simulations to demonstrate the benefits available when using complex averaging and then develop an algorithm for performing complex averaging of in-vivo cDTI data, which accounts for the induced phase variations.

\section{Methods}

To compare the performance of magnitude and complex averaging in the presence of noise, simulated noisy diffusion weighted images of the left ventricle were calculated with b-values from $50-3000 \mathrm{smm}^{-2}$ based on previously published in-vivo cDTI data[2]. Magnitude or complex averaging was performed before processing the images with the software we typically use for in-vivo cDTI data.

Additionally, in-vivo cDTI was performed on a Siemens Skyra in 10 subjects using the STEAM-EPI sequence[3] with 4 b-values, $500-2000 \mathrm{smm}^{-2}, 12$ averages, 6 directions, SENSE reconstruction (magnitude and phase images) and $2.8 \times 2.8 \times 8 \mathrm{~mm}^{3}$ resolution. In order to remove motion-induced phase, each image (Real+Imaginary) was filtered (pyramid mask in k-space, extent: $1 / 4$ field-of-view [4]) and the phase of the low-resolution copy was

${ }^{1}$ Cardiovascular Biomedical Research Unit, The Royal Brompton Hospital, London, UK

Full list of author information is available at the end of the article removed from the original images. The images were then processed as for the simulations.

\section{Results}

Figure 1 shows the results of simulations demonstrating the errors in mean diffusivity (MD), fractional anisotropy (FA) and helical angle (HA) for a range of b-values. At low b-values, no benefit from complex averaging is observed, but at high b-values $>1000 \mathrm{smm}^{-2}$, the underestimation of MD and FA using magnitude averaging ("squashing the peanut"[5]) can be compensated for by using complex averaging.

Figure 2 compares results obtained in vivo, processed with magnitude and complex averaging. As predicted by the simulations, FA and MD are increased when using complex averaging and changes in the parameter maps are evident.

\section{Conclusions}

Large b-values are required to avoid under-estimation of FA and MD in CDTI. We have demonstrated that the phase introduced by diffusion weighting in vivo can be corrected for within the reconstruction, allowing us to partially compensate for the noise-floor effects present at high b-values by using complex averaging.

\section{Funding}

This work was performed at the National Institute for Health Research Funded Cardiovascular Biomedical Research Unit at The Royal Brompton Hospital and Imperial College.

\section{Authors' details}

'Cardiovascular Biomedical Research Unit, The Royal Brompton Hospital, London, UK. ${ }^{2}$ National Heart and Lung Insitute, Imperial College, London, UK. 

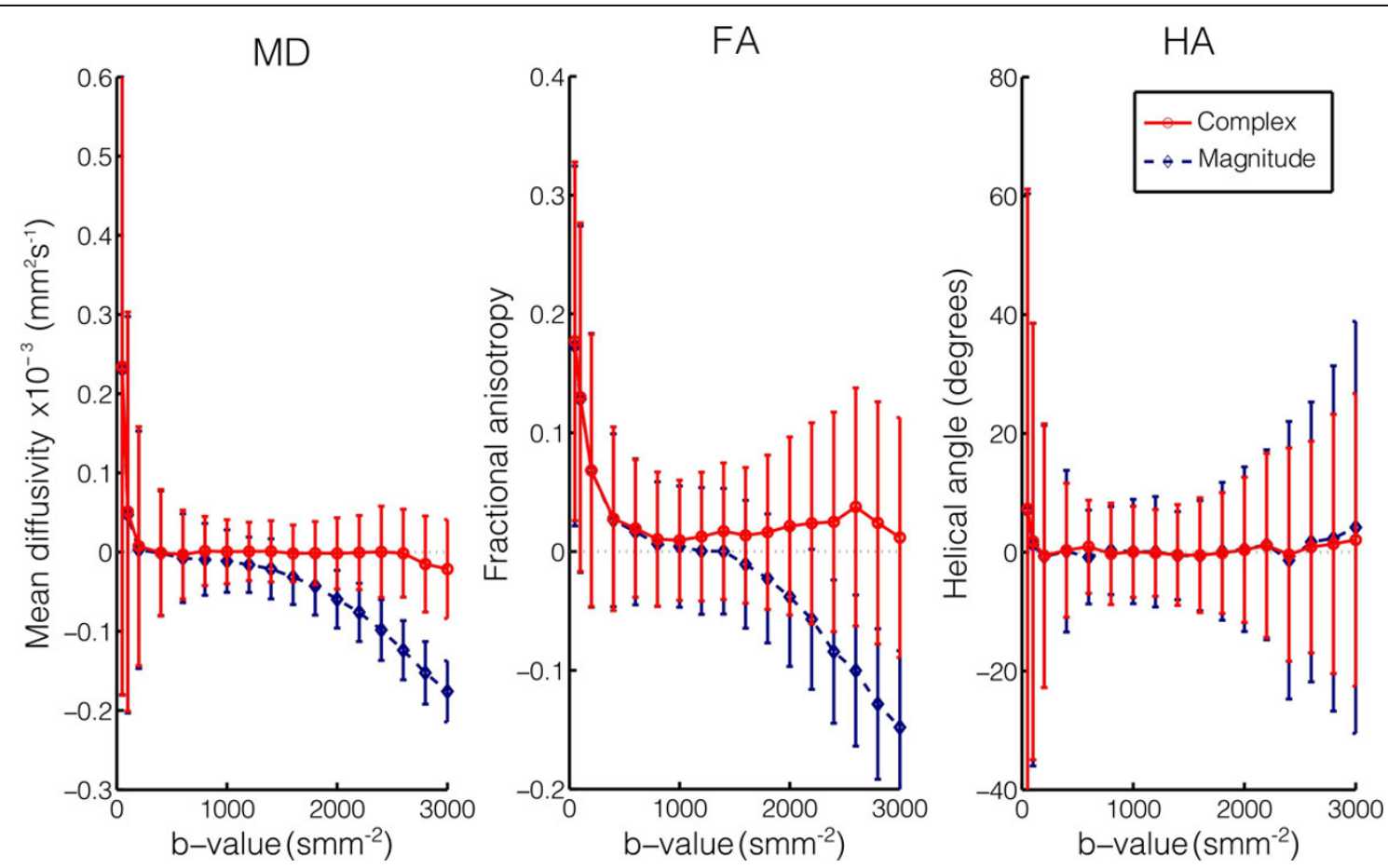

Figure 1 Simulations showing the mean difference \pm standard deviations in CDTI derived parameters (mean diffusivity: MD, fractional anisotropy: FA, helical angle: HA) from the ground truth with a range of b-values. The simulated SNR was 17 in the unaveraged reference images and 11 averages were used. The error is calculated as [simulated result] - [ground truth] so a positive bias indicates an over-estimation of the parameter.
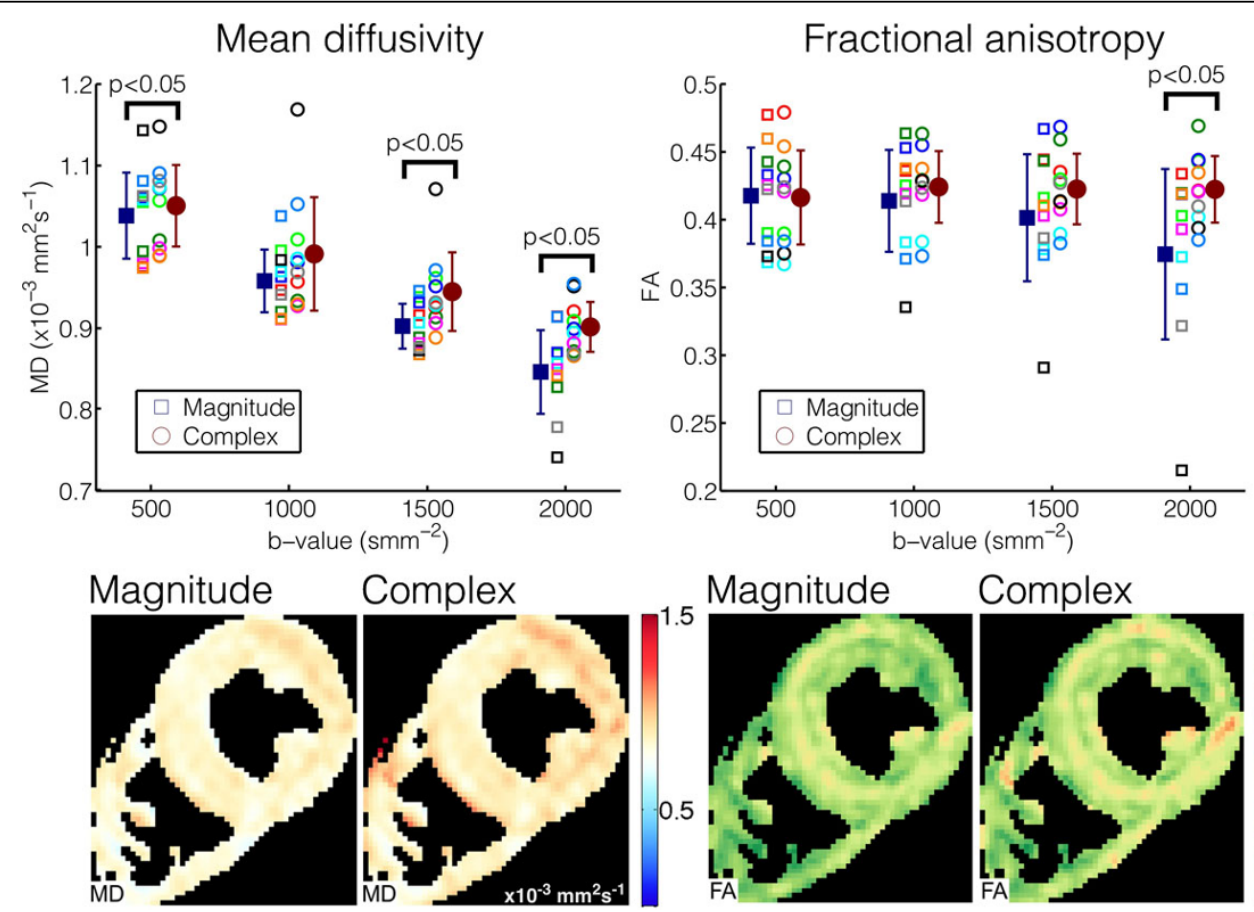

Magnitude

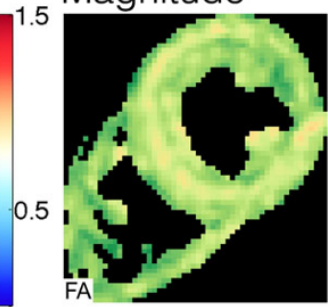

Complex

Figure 2 In-vivo results showing the mean diffusivity and fractional anisotropy for all 10 subjects (upper plots) at b=500, 1000, 1500 and $2000 \mathrm{smm}^{-2}$. Results at each b-value were compared with a paired t-test and significant results are indicated on the plots. Example mean diffusivity and fractional anisotropy maps from one subject are also shown from data acquired with $b=2000$ smm $^{-2}$, highlighting the recovery of lost MD and FA at high b-values when the complex averaging algorithm is used. 
${ }^{3}$ Division of Transmural Research - National Heart Lung and Blood Institute, National Institutes of Health, Bethesda, MD, USA.

Published: 3 February 2015

\section{References}

1. Skare: ISMRM 2009, 1409.

2. Scott: MRM 2014, DOl:10.1002/mrm.25418.

3. Nielles-Vallespin: MRM 2012, DOl:10.1002/mrm.24488.

4. Pipe: MRM 2002, DOI:10.1002/mrm.10014.

5. Jones: MRM 2004, DOl:10.1002/mrm.20033.

doi:10.1186/1532-429X-17-S1-038

Cite this article as: Scott et al:: Improving the accuracy of cardiac DTI by averaging the complex data. Journal of Cardiovascular Magnetic

Resonance 2015 17(Suppl 1):O38.

Submit your next manuscript to BioMed Central and take full advantage of:

- Convenient online submission

- Thorough peer review

- No space constraints or color figure charges

- Immediate publication on acceptance

- Inclusion in PubMed, CAS, Scopus and Google Scholar

- Research which is freely available for redistribution

Submit your manuscript at www.biomedcentral.com/submit
C) Biomed Central 